\title{
Les espaces du naturisme : modèle allemand et exception française?
}

Spaces of naturism: a German model and French exception?

Die Räume der Freikörperkultur : ein deutsches Modell und die Besonderheit

Frankreich?

Emmanuel Jaurand

\section{OpenEdition}

\section{Journals}

Édition électronique

URL : http://journals.openedition.org/rge/818

DOI : $10.4000 /$ rge. 818

ISSN : 2108-6478

Éditeur

Association des géographes de l'Est

Édition imprimée

Date de publication : 1 janvier 2007

ISSN : 0035-3213

Référence électronique

Emmanuel Jaurand, "Les espaces du naturisme : modèle allemand et exception française ? 》, Revue Géographique de l'Est [En ligne], vol. 47 / 1 | 2007, mis en ligne le 22 décembre 2009, consulté le 07

septembre 2020. URL : http://journals.openedition.org/rge/818 ; DOI : https://doi.org/10.4000/rge.818

Ce document a été généré automatiquement le 7 septembre 2020

Tous droits réservés 


\title{
Les espaces du naturisme : modèle allemand et exception française?
}

\author{
Spaces of naturism: a German model and French exception? \\ Die Räume der Freikörperkultur : ein deutsches Modell und die Besonderheit \\ Frankreich?
}

Emmanuel Jaurand

\section{Introduction}

1 Si la nature n'a rien de spécifiquement géographique, il se trouve que les géographes s'y sont largement intéressés, et que la façon d'appréhender la nature concourt largement à caractériser le paradigme géographique dominant à une certaine époque. A l'approche naturaliste des milieux naturels, caractéristique de la géographie française classique, s'est largement substituée une approche environnementale centrée sur la modification et la préservation des milieux par les sociétés humaines (Robic, 1992). A ces questions fondamentales de l'impact des sociétés humaines sur la nature, s'ajoutent celles du regard que les civilisations portent sur la nature, regard qui est à l'origine de représentations, de pratiques spatiales, voire de la production de territoires.

Dans le cas de l'Allemagne, évoquer le naturisme relève encore moins qu'ailleurs d'une démarche anecdotique ou anodine. Ce phénomène de civilisation caractéristique de l'Allemagne et plus largement, de l'Europe du nord, repose sur une conception mythique de la nature, vue comme bienfaisante et incarnation de l'œuvre divine, et se traduit par une pratique du nu collectif (nudisme) dans des lieux permettant un contact direct avec un environnement "naturel », en tout cas apparemment peu artificialisé. Avant même l'essor de l'écologie politique, le mouvement naturiste insistait sur le besoin de nature et les limites des ressources de la terre, thèmes dominants du 12e congrès de la Fédération Naturiste Internationale (1970) consacré à «l'homme et son milieu de vie». En 1974, le congrès d'Agde adoptait la définition suivante du naturisme: «un mode de vie en harmonie avec la nature, exprimé dans une nudité 
communautaire, pratiquée dans le respect de soi, des personnes ayant des opinions différentes et de l'environnement" (FNI, 2003, p. 8). Dans le naturisme, la pratique nudiste est indissociable d'une certaine représentation de la nature et de la place que l'homme doit y prendre, renvoyant à des mythes fondateurs tels le jardin d'éden.

Depuis les années 1990 la géographie française connaît un "tournant culturel», largement marqué par les apports de la «New Cultural Geography» anglo-saxonne (Claval, 1995), moins préoccupée par les techniques de la vie sociale que par l'interprétation symbolique que les sociétés se font de leur environnement. Ainsi, l'analyse des discours, des représentations voire des mythologies propres à des groupes comme les naturistes permet de comprendre la façon dont ils ressentent la nature, vivent le monde et pratiquent l'espace. Mais convaincus de l'intérêt d'une approche géographique globale qui dépasse les barrières d'école ou de méthode, nous souhaitons nous appuyer à la fois sur les témoignages subjectifs mobilisés par la géographie culturelle et d'autres données classiques en géographie du tourisme et des loisirs telles les statistiques sur les lieux naturistes, dont l'intérêt nous semble aussi partiel qu'irremplaçable. Notre objectif est de comprendre à la fois les pratiques collectives et les structures spatiales du naturisme allemand, en distinguant les espaces fermés du naturisme, clubs et centres avec terrain et accès contrôlé, (I) et les espaces ouverts à tout public que sont les plages et les parcs (II). Enfin, l'étude des dynamiques spatiales contemporaines permettra d'envisager les conséquences de la réunification et du développement du tourisme international sur le «modèle naturiste allemand» (III). Une comparaison avec la France, autre grand pays du naturisme mondial, permettra de mieux saisir le caractère «modèle" de la situation allemande, fondée sur un enracinement profond de la pratique nudiste et de l'attachement à la nature.

\section{Les deux premiers pays naturistes du monde}

Se voulant un mouvement de civilisation, le naturisme dispose aussi d'organisations fédérales et d'établissements à l'abri des regards d'autrui qui lui permettent de trouver un double ancrage institutionnel et spatial. Ce naturisme officiel est aussi développé en France qu'en Allemagne, mais avec des logiques de répartition spatiale qui révèlent des conceptions et des pratiques différentes du naturisme.

\section{A. Structures et effectifs du naturisme}

5 La fédération allemande de naturisme fondée en 1918 (F.K.K., Freikörperkultur, littéralement Culture du Corps Libre devenue D.F.K., Deutscher Verband für Freikörperkultur), est la plus ancienne du monde. Avec la Fédération Française de Naturisme, née en 1950, elles ont été jusqu'à ces dernières années les deux plus puissantes composantes du naturisme international (Fédération Internationale de Naturisme, 1953) en terme d'adhérents. Une récente tendance à la baisse a fait passer la D.F.K. derrière la fédération néerlandaise (62 000 membres en 1995 et 50000 en 2003 pour la D.F.K. contre 70000 membres en 2005 pour la seconde, d'après les sites internet de la FNI et de Vivre nu), et a vu la Fédération Française de Naturisme se diviser et perdre les $2 / 3$ de ses effectifs (maximum de 83500 en 1983, moins de 25000 en 2005). Mais le déclin du militantisme naturiste ne remet pas en cause la reconnaissance du rôle du mouvement en faveur de la jeunesse et de l'environnement par les pouvoirs 
publics. Surtout, la fin de l'obligation de possession d'une licence fédérale pour accéder aux structures naturistes (sauf exceptions) a permis d'élargir le public potentiel.

6 Celui-ci est en effet infiniment plus nombreux que ne le laissent entrevoir les effectifs des fédérations, même si les statistiques disponibles révèlent une grande marge d'incertitude. En Allemagne, ceux qui fréquentent les structures naturistes fermées et ceux qui pratiquent régulièrement le nudisme en espace public sont estimés à un nombre total compris entre 8 et 12 millions de personnes (fédérations allemande, québécoise et internationale de naturisme), soit 10 à $15 \%$ de la population totale : ce pourcentage est égalé aux Pays-Bas voisins (1,9 million de pratiquants pour 16,3 millions d'habitants). En France, les estimations concernant les clients des centres de vacances naturistes varient entre 1 et 1,5 million de personnes (Barthe, 2001), tandis que d'après une enquête de l'IFOP de 1993, le total des naturistes réguliers et les nudistes occasionnels s'élèverait à 5,5 millions. De telles valeurs, qui tiennent plus de l'ordre de grandeur que de la statistique exacte, montrent que le naturisme et le nudisme sont plus que des pratiques sociales marginales dans les deux pays.

Cette forte implantation est assurée par les structures de loisirs et d'hébergement naturistes. Alors que 850 sites naturistes disposant d'un terrain existent dans le monde (site internet Nacktbaden), le guide de la Fédération Naturiste Internationale en recense 170 pour la France et 141 pour l'Allemagne (FNI, 2003), qui s'affirment ainsi comme les deux premiers pays naturistes du monde. Ces centres avec terrain permettent la pratique de la nudité collective en plein air pour quelques heures, le week-end ou de longs séjours dans le cas des centres de vacances. Ils disposent d'équipements sportifs et, le cas échéant, de formes d'hébergement variées (tentes, mobile-homes, bungalows...), de commerces et de services permettant d'effectuer tous les actes de la vie courante dans un état de nudité, ce qui fonde le mode de vie naturiste. Ces centres sont conçus comme des lieux permettant de « vivre en harmonie avec la nature », selon le slogan du centre F.K.K. de l'île d'Amrum (Schleswig-Holstein). Un tel objectif passe par le contact le plus souvent possible avec les éléments naturels à l'occasion d'activités sportives (natation, randonnée nue, vélo...), une alimentation saine, le respect des rythmes biologiques (silence nocturne) et des normes environnementales dans l'aménagement du centre (absence de circulation automobile dans le centre du fait de l'isolement et de l'éloignement du parking, priorité à l'énergie solaire...). Promouvant un tourisme durable, une majorité des centres naturistes adhère à des chartes d'environnement et mène des actions de sensibilisation des clients en faveur d'une réduction des productions des déchets et des consommations d'eau et d'énergie (Barthe-Deloizy, 2003). Ces préoccupations environnementales ont d'abord touché les centres naturistes allemands à l'image de leurs homologues d'autres pays germaniques (Danemark, Autriche, Suisse) : ce trait marquant du modèle naturiste de l'Europe germanique s'est développé en France depuis la fin des années 1990 (éco label Clefs Vertes).

\section{B. Des logiques de répartition interne différentes}

8 Alors que France et Allemagne offrent des sites naturistes dont le nombre et le souci écologique sont pratiquement identiques, une comparaison de leur répartition spatiale révèle des différences dans la pratique naturiste (fig. 1). 
Figure 1 : Répartition des centres naturistes et des plages nudistes officielles en Allemagne et en France.

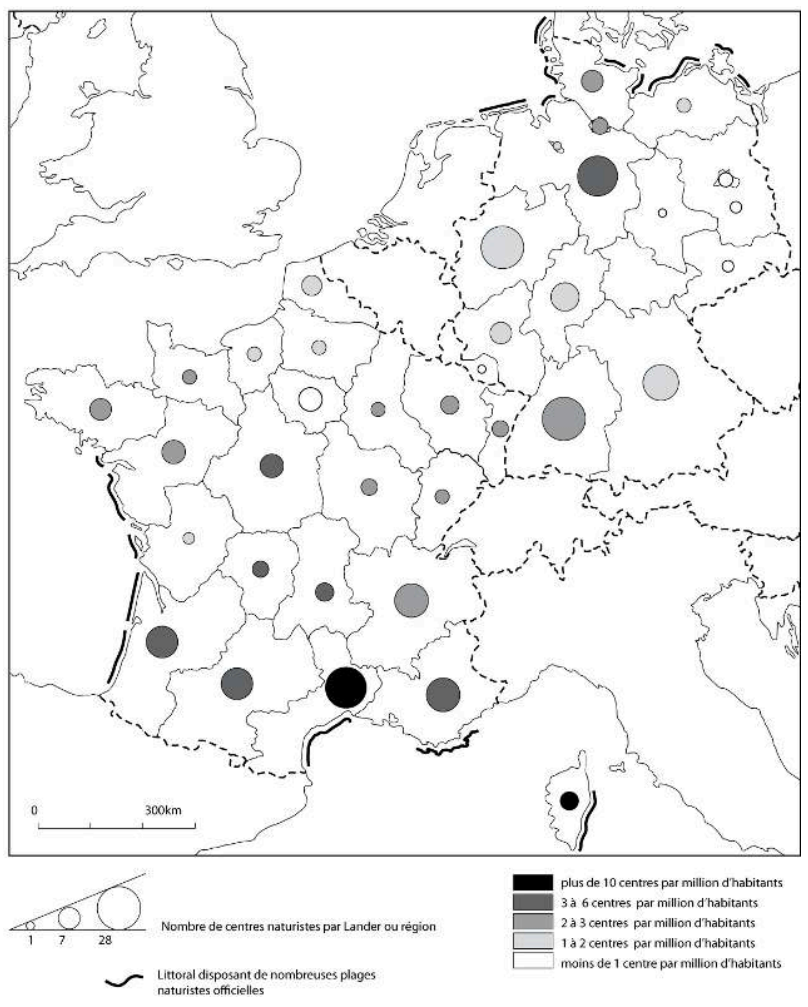

9 La géographie du naturisme allemand offre un premier contraste de part et d'autre de la frontière qui a divisé le pays de 1949 à 1989. Les 6 Länder correspondant à l'ex-RDA n'abritent en effet que 11 centres naturistes avec terrain contre 130 pour l'ex-Rfa, ce qui correspond à des proportions respectives de 2 centres pour 3 millions d'habitants et 1 million d'habitants. Il s'agit là d'un héritage lié à l'histoire différente du naturisme sous les 2 régimes. En RDA, le naturisme a été officiellement interdit dès les débuts du régime pour des raisons idéologiques (assimilation au nazisme et à la décadence impérialiste), et il ne pouvait de toute façon exister d'association privée ou d'entreprise commerciale; mais ceci ne signifie évidemment pas une absence de pratique du nu collectif sous des formes non encadrées, comme l'atteste la permanence depuis l'aprèsguerre d'un nudisme de plage fort répandu sur les côtes de la RDA. En RFA, des associations naturistes se sont reconstituées après-guerre en rejetant les dogmes qui avaient été imposés au mouvement sous le nazisme, en particulier le principe de sélection raciale; le naturisme ouest-allemand s'est structuré avec l'accord des pouvoirs publics et s'est intégré au naturisme international.

Il est remarquable qu'au sein des anciens Länder, la répartition des centres naturistes est globalement moins hétérogène qu'à l'échelle de toute l'Allemagne et aussi qu'en France. On peut y voir un avatar du polycentrisme allemand. En effet, le mouvement naturiste allemand qui s'est constitué dans le premier tiers du XXe siècle, s'est développé à partir de plusieurs foyers urbains sur la base d'initiatives individuelles (Toepfer, 1997). Les hauts lieux historiques du naturisme allemand ont été tour à tour Berlin, Stuttgart, Hambourg, Munich et Leipzig (Cluet, 1999 et comm. écrite), sans effacement des centres plus anciens. Cette géographie historique et actuelle du naturisme allemand est indifférente au clivage religieux entre le nord majoritairement protestant et le sud majoritairement catholique, clivage qui souffre d'ailleurs 
d'exceptions à grande échelle (cas du Münsterland d'une part, ou de la Franconie d'autre part). Certes, il existe bien un léger avantage des Lander les plus septentrionaux en terme de ratio centre naturiste/nombre d'habitants, de la Basse-Saxe au Mecklembourg-Poméranie occidentale. Ceci nous semble plutôt lié à l'importance du tourisme, d'orientation presque exclusivement nationale (moins de $10 \%$ d'étrangers parmi les touristes, Boyer, 2000) : de nombreux centres sont implantés en bord de mer ou de lac et reçoivent une clientèle allemande à la fois locale et extra-régionale.

11 En France, le trait marquant de la géographie naturiste réside dans un fort gradient latitudinal en faveur du sud, de l'Aquitaine à la Corse. Le Languedoc-Roussillon apparaît comme la région record avec ses 25 centres, soit une proportion d'un centre pour 100000 habitants, 6 fois plus forte que celle de toute l'Allemagne. La méridionalité prononcée du naturisme français est encore plus éclatante si l'on s'intéresse aux capacités d'accueil des centres : le seul département de la Gironde rassemble $30 \%$ de la capacité d'hébergement en centre naturiste de toute la France! L'héliotropisme du naturisme français rappelle, en plus accentué, celui du tourisme estival en général. Une nuance spécifique du naturisme existe cependant, y compris à l'échelle de chaque région du sud: les régions et secteurs littoraux d'implantation préférentielle sont ceux qui ont été mis en tourisme tardivement (Aquitaine, sauf la côte basque, LanguedocRoussillon, côte varoise et Corse orientale). Le naturisme s'y est développé à partir des années 1960-70 de façon concomitante à l'offre touristique, sans trop de conflit d'usage et sans problème d'image.

\section{Des conceptions différentes et complémentaires du naturisme : une pratique culturelle de nature en Allemagne, un segment touristique en France}

12 Les différentes logiques de répartition des centres naturistes en Allemagne et en France renvoient en fait à des différences dans la conception et la pratique naturistes.

13 En Allemagne, si l'on fait abstraction de l'exception des nouveaux Länder, la répartition assez homogène des centres naturistes par rapport à celle de la population résidente traduit une offre naturiste de proximité, même en dehors des vacances estivales. Le naturisme est en fait conçu comme une pratique culturelle intégrée à la vie quotidienne et réalisée par une population citadine, à l'occasion des temps de loisirs en toutes saisons. En témoigne le fait qu'outre les clubs naturistes urbains sans terrain, la majorité des centres naturistes sont situés à la périphérie immédiate des agglomérations urbaines, et sont donc accessibles pour y passer une partie de la journée ou un week-end. Si l'on prend le cas du land de Rhénanie-du-Nord-Westphalie, qui n'est guère touristique, la plus forte concentration de centres correspond exactement à sa partie la plus urbanisée : le triangle Duisburg-Bonn-Dortmund abrite 16 des 27 centres naturistes du Land, la plupart des autres se localisant à proximité immédiate de villes comme Aachen (3) ou Bielefeld (2) : les centres situés dans l'espace rural isolé font figure d'exception. En France au contraire, les centres naturistes des périphéries urbaines sont rares; la majorité d'entre eux se trouvent dans des régions touristiques, en bord de mer ou à la campagne, et s'apparentent davantage à des centres de vacances essentiellement animés en été. Le basculement du naturisme dans le secteur touristique estival a été plus précoce et complet en France, dès les années 1930 (Baubérot, 2004). En Allemagne, l'apparentement du naturisme à une pratique 
culturelle de loisir est attesté par le fait que nombre de piscines publiques y proposent de longue date des créneaux horaires réservés aux naturistes, alors que le phénomène est récent et limité en France. Une photographie datant de 1929 montre des nudistes hommes et femmes dans une piscine découverte près de Francfort (Ross, 2005, p. 37). Et J.-L. Bouland (1997, p. 22) rapporte qu'en 1960, le conseil municipal de Sarrebruck qui avait annulé une autorisation d'utilisation de la piscine municipale pour des réunions "gymniques ", a été condamné et a dû revenir sur sa décision, le tribunal estimant que le naturisme repose sur des principes d'éthique.

14 Certes, le naturisme allemand relève partiellement du secteur touristique. Dans les deux pays, les centres de vacances ont suivi les mutations et améliorations de l'hébergement touristique en accord avec les exigences croissantes en confort de la clientèle : les «camps » des naturistes militants de l'après-guerre (ainsi appelés parce que l'hébergement se faisait dans des tentes) ont laissé la place à des campings équipés de piscines, de restaurants et autres commerces, et proposant également des logements en bungalows ou mobile-homes. En Allemagne et surtout en France, des domaines résidentiels naturistes en dur, assimilés à des villages-vacances spécialisés (LozatoGiotart, 1993) proposent des prestations et des activités haut de gamme (sauna, jacuzzi, thalassothérapie...). L'exception française réside surtout dans la conception des quartiers urbains naturistes du Cap d'Agde et de Port-Leucate, sur la côte languedocienne : l'intégration du tourisme naturiste à la politique d'aménagement du territoire et la construction de véritables stations balnéaires naturistes sont sans équivalent en Allemagne. Les centres naturistes y sont restés l'œuvre exclusive d'associations ou d'entrepreneurs privés, et se caractérisent par une faible densité d'occupation, la dominance de l'hébergement en toile ou en caravane, davantage en accord avec le principe de recherche de la proximité de la « nature».

Enfin, l'origine de ceux qui fréquentent les centres naturistes achève de distinguer les cas allemand et français. En Allemagne, la clientèle des centres naturistes est essentiellement allemande, y compris en été : le tourisme international y est surtout tourné vers les villes et les sites historiques, tandis que les espaces de nature, notamment littoraux, attirent le tourisme autochtone (Boyer, 2000). Les rivages de la mer du Nord et de la Baltique, avec moins de 1500 heures d'ensoleillement et une eau réputée à tort fraîche, séduisent peu les étrangers. Bien différente est la situation de la France en Europe occidentale, qui tient à la fois du carrefour et d'un sud relativement proche; la croissance de l'offre d'hébergement naturiste soutenue par l'Etat a transformé la France en première destination naturiste mondiale. Les étrangers y représentent $52 \%$ des clients des centres naturistes, et parmi eux, on trouve $34 \%$ de néerlandais, $28 \%$ d'Allemands, $19 \%$ de Belges, $10 \%$ de Britanniques et $6 \%$ de Suisses (Espaces, 1999, p. 22). Pour les Allemands notamment, la France permet ainsi une pratique naturiste vacancière complémentaire de celle qu'ils ont chez eux le reste de l'année. 
Photo 1 : Les bords de l'Isar en plein centre de Munich. On notera la cohabitation de nudistes et de "textiles». Juillet 2006

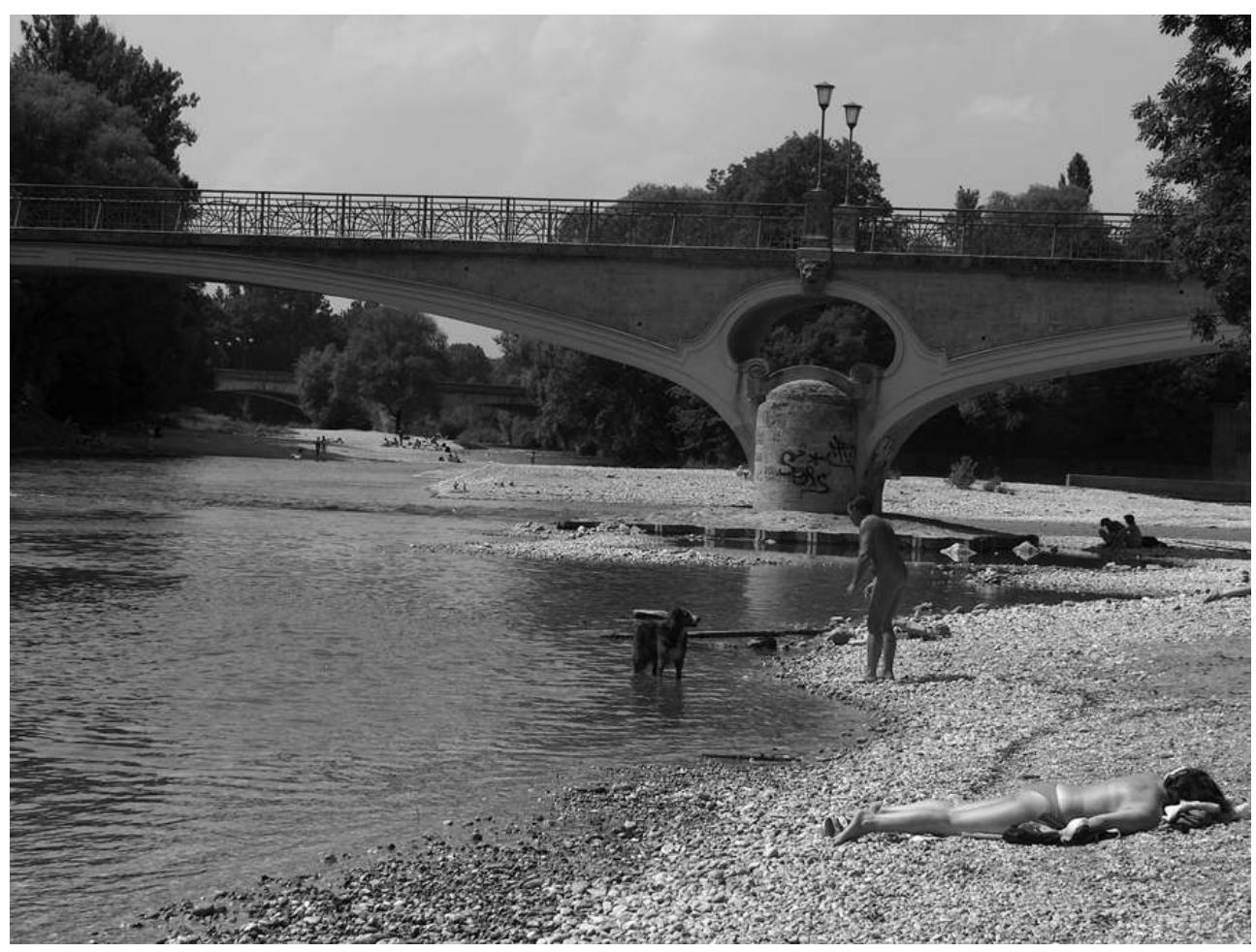

\section{Le nudisme en espace public de nature : banalisation en Allemagne, confinement en France}

Dans les centres naturistes, et dans les piscines à certaines heures, la nudité collective est enclose et de règle, ce qui pourrait faire croire à l'existence d'une étanchéité avec le reste du monde, celui de la nudité interdite que les naturistes qualifient de «textile». Or il existe, de façon singulièrement puissante et caractéristique dans le modèle allemand, un nudisme « hors les murs », en espace public de plein air, donc accessible à la présence et aux regards d'autrui.

\section{A. Le nudisme tranquille des espaces de nature en ville, spécificité du modèle allemand}

Dans sa thèse d'histoire sur le nudisme allemand, M. Cluet (1999) rapporte que de passage à Berlin en 1930, Giraudoux notait avec étonnement l'invasion des lacs et parcs par des jeunes dévêtus. Cette pratique sociale bien particulière, laissant encore nombre d'étrangers médusés, explique le titre d'un ouvrage sur l'Allemagne de l'entre-deuxguerres : Au pays des hommes nus (L.C. Royer, 1929). Ainsi que le montre le film de Robert Salis (1998), le fait est que dans la plupart des grandes villes allemandes, de Berlin à Munich en passant par Francfort, on note à la belle saison et en particulier à l'heure de la pause-déjeuner ou l'après-midi, la présence de nudistes bronzant dans des espaces verts ou sur les rives des lacs et cours d'eau. Nous avons pu nous-même constater une telle pratique au Tiergarten de Berlin, bois situé en cœur de ville, et en plusieurs endroits de Munich en juillet 2006. Dans ce dernier cas, les nudistes se 
rassemblaient soit en un secteur de l'Englischer Garten, soit sur les bancs de galets des bords de l'Isar, au contact du cœur historique de la ville et à la vue des passants empruntant les ponts ou les quais (photo 1). Fait significatif de la banalité du nu, les nudistes se trouvaient mêlés à de nombreuses personnes vêtues de maillots, sans qu'il n'y ait de regards appuyés ou de propos moqueurs.

C'est cette nudité publique urbaine décomplexée et tranquille qui nous semble le trait le plus original du modèle naturiste allemand. Il serait plus exact de parler de modèle germanique dans la mesure où le même phénomène se retrouve au Danemark, en Suède, en Finlande, aux Pays-Bas... Dans ces pays, et sous des formules variables, la nudité intégrale est permise en tout lieu «naturel » dans la mesure où la personne nue n'importune pas les voisins et passants par des gestes déplacés. Le nu public n'y est donc pas considéré comme incongru dans des espaces de "nature» (parcs, bords de fleuve, lac ou mer). Si un nudiste, à l'attitude non exhibitionniste ou provocatrice, se trouvait dans un lieu où l'absence de vêtement paraîtrait déplacée, il risquerait un simple rappel à l'ordre assorti de l'injonction d'aller ailleurs. Un autre aspect de la relation forte entre nu et «nature » est la reconnaissance explicite ou implicite d'un droit «naturel » à la nudité, formellement reconnu depuis 1996 par la législation néerlandaise à la demande de la fédération naturiste nationale.

Cette pratique de la nudité en public, détachée de toute connotation sexuelle, est même antérieure au développement du mouvement naturiste (König, 1990). Les baignades collectives nues dans les lacs ou la mer étaient une pratique courante sous l'Empire allemand, dans des endroits isolés pour ne pas tomber sous le coup de la loi. Ce nudisme « invisible » était considéré avec bienveillance ou indifférence, en particulier dans le nord protestant. Précisément, une explication du modèle germanique de nudité publique est religieuse. J. Viard (1990) souligne l'intérêt prononcé du monde protestant pour la nature : en l'absence de corps intermédiaire entre Dieu et lui, «le fidèle ne pourra vérifier la gloire (du Créateur) que dans cette création qui l'entoure ». Cette jonction du vieux fonds panthéiste et de la religion réformée permet de voir dans le nudisme en espace naturel, une manifestation de rapprochement et de célébration de l'œuvre divine, renvoyant aussi au mythe du jardin d'éden, référence majeure de la nudité innocente, c'est-à-dire vécue sans honte. La vision catholique de la nature est plus empreinte de méfiance et de volonté d'assujettissement (Pitte, 1999). Mais majoritaire à l'échelle nationale, le protestantisme a aussi pu imprégner les pratiques culturelles des catholiques allemands (Boyer, 1996); et ce d'autant plus que la nudité collective est présente chez les peuples germaniques depuis l'Antiquité (tradition du combat nu). Il est ainsi difficile de distinguer les parts respectives de l'ethnicité et de la religion dans l'enracinement du nudisme allemand.

\section{B. Le nudisme déborde très largement des plages homologuées F.K.K.}

Entièrement situé dans des régions protestantes, le littoral allemand montre un développement du nudisme balnéaire typique de l'Europe du nord, tant dans les anciens que dans les nouveaux Länder. Le nudisme est même pratiqué par une majorité d'estivants en certains secteurs, en particulier dans les îles (des îles Frisonnes à Rügen). Ainsi, sur l'île de Sylt située à la frontière avec le Danemark, $70 \%$ des 300000 touristes pratiqueraient le nudisme (Wedemeyer, 2001). Il est vrai que sur cette île peu urbanisée 
qui offre des plages et des dunes sans interruption sur $40 \mathrm{~km}$, l'espacement et le caractère « naturel » de la côte aident au déploiement de la pratique nudiste. Elle y est attestée dès les années 1850 , sur recommandation médicale pour soigner les maladies de peau; des habitants avaient aussi pris l'habitude de se baigner nus, relayés à la fin $\mathrm{du}$ XIXe siècle par des artistes en villégiature, puis par des estivants. C'est dire l'ancienneté du nudisme balnéaire et la variété des publics concernés. La banalité de cette pratique pour l'opinion est d'ailleurs révélée par 2 sondages des années 1980, selon lesquels $68 \%$ des allemands approuvent ou tolèrent la baignade sans maillot (site internet de la fédération québécoise de naturisme).

21 La banalité sociale du nu balnéaire s'exprime dans l'espace par la visibilité et l'institutionnalisation des plages nudistes. Les plages homologuées FKK figurent comme telles sur les cartes topographiques ou le Bild Atlas, les brochures et plans des offices de tourisme, sont signalées au public par des panneaux, et des arrêts de bus portent même l'appellation FKK-Strand! Ces plages nudistes sont aussi équipées que les autres: la plage FKK de List, au nord de Sylt, dispose de toilettes, douches, tables de pique-nique, et des fameux fauteuils en corbeille numérotés... (photo 2). Des activités sportives nues y sont proposées, comme le volley-ball, et à heures régulières, des cours de gymnastique. Si ces plages FKK ne se trouvent pas au cœur des stations balnéaires, beaucoup n'en sont guère éloignées. A Travemünde, la plage FKK célèbre avant 1989 pour être à portée de fusil et de jumelles des soldats est-allemands, se situe à une dizaine de minutes à pied du centre animé ; elle partage son poste de surveillance avec la plage « textile» voisine.

Photo 2 : La plage nudiste officielle (F.K.K.) de List, au nord de l'île de Sylt (Schleswig-Holstein). Août 2004.

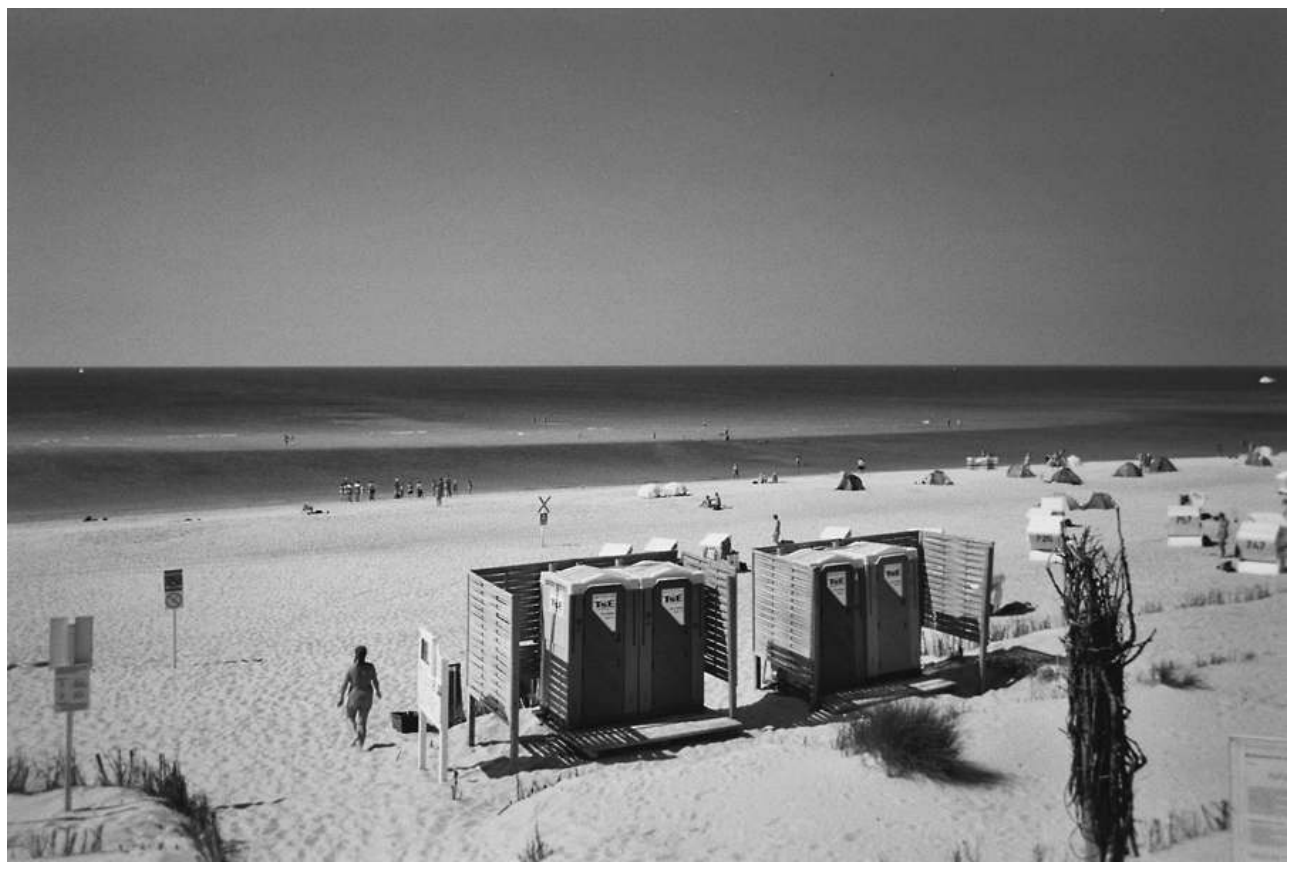

Si la nudité est globalement bien respectée sur ces plages officielles, elle existe également sur d'autres plages. Il peut s'agir de plages situées en continuité avec les plages F.K.K. : au-delà du panneau F.F.K. Ende s'observe un mélange entre les nudistes et les « textiles », ce qui relève d'un phénomène de diffusion. Ainsi à Westerland (Sylt), 
la plage $n^{\circ} 55$ comprise entre deux plages F.K.K. est très majoritairement occupée par les nudistes, qui ne se soucient guère des limites officielles. Ou bien, il s'agit de plages isolées, éloignées de toute zone urbaine, où le nudisme est pratiqué de façon sauvage par un public disséminé sur des kilomètres de côte vierge de constructions. On peut citer l'exemple d'une grande plage située à l'est de Rostock. Un vaste secteur investi par les nudistes se déploie sur plus de $5 \mathrm{~km}$ entre les stations de Markgrafenheide et GraalMüritz et s'adosse à une zone naturelle protégée marécageuse (Naturschutzgebiet) ; la situation de marge répond à la recherche de l'entre-soi nudiste, loin des plages textiles bondées des stations, dans un cadre correspondant à une attente de "nature sauvage ». Un dernier indice de l'intégration spatiale du nudisme balnéaire est sa présence sur pratiquement toute la façade littorale allemande, jusque dans les secteurs les plus réputés ou huppés (îles de Rügen et de Sylt), ce qui signe une différence avec le littoral français (fig. 1).

\section{En France, un nudisme en espace public limité à des plages isolées}

23 Alors que les premières autorisations de la pratique du nudisme sur les plages remontent aux années 1920 en Allemagne, il faut attendre 1956 à Montalivet et les années 1970-80 pour la plupart des autres plages nudistes françaises. C'est dire qu'en France, le nu a d'abord été enfermé dans des centres (Centre Hélio-Marin de Montalivet, 1950) pour des raisons d'incompatibilité avec la loi et les mentalités, avant d'investir certaines portions bien délimitées et généralement isolées de l'espace balnéaire. Par arrêté municipal, la pratique du nudisme y est autorisée ou tolérée, ce qui constitue une exception à l'article 330 du code pénal de 1994 qui a vu la notion d'» exhibition des caractères sexuels imposés à la vue d'autrui » se substituer à celle d'» outrage public à la pudeur ». Il est significatif qu'en France, le nudisme sauvage en espace naturel tombe sous les coups de la loi, et soit punissable d'une peine allant jusqu'à un an d'emprisonnement, alors qu'en Allemagne ce sont les attitudes restrictives à l'égard du nudisme qui sont condamnées : la jurisprudence libérale des années 1920, qui déboutait les plaignants qui s'offensaient d'une nudité dépourvue de dimension sexuelle, a été invoquée par certains tribunaux sous le régime nazi, contre la législation répressive de 1933 ! C'est dire la différence de mentalité, et d'attitude de la société à l'égard du nu public entre les deux pays.

De fait, en France, le nudisme sauvage est pratiqué sur des plages protégées des regards d'autrui par leur accès long ou difficile, et leur absence de signalisation hors de publications confidentielles ou de sites internets spécialisés. Entre une tolérance de fait et une répression par les forces de gendarmerie et de police, les situations locales varient d'une commune à l'autre, mais globalement, le nudisme sauvage est plus mal considéré sur les littoraux de tourisme ancien et aisé (Côte d'Azur, Côte Fleurie...) (Jaurand et de Luze, 2004). Si le nudisme, autorisé ou sauvage, est également pratiqué sur des plages « vertes » (en bord de lac ou de cours d'eau), il reste interdit et rarissime dans des espaces verts urbains, ce qui distingue la France du modèle germanique. En Ile-de-France, seuls des secteurs confinés de bois (Vincennes, Verrières) et des rives de Marne accueillent occasionnellement quelques nudistes, très peu nombreux et majoritairement gays, exposés au risque permanent de verbalisation. Il est vrai que pour les autorités, la dénudation publique en ville n'est guère à l'ordre du jour, comme 
en témoigne l'arrêté de la municipalité parisienne interdisant les seins nus à ParisPlage l'été 2006...

\section{Dynamiques spatiales du nudo-naturisme : des clivages nationaux entre effacement et permanence?}

Les mutations sociales voire les événements politiques interfèrent avec les évolutions propres au mouvement naturiste et à la pratique nudiste. Plusieurs dynamiques spatiales du naturisme en cours ces dernières années et décennies à différentes échelles montrent ainsi un effacement relatif des spécificités nationales: qu'est devenu le modèle naturiste allemand sous l'effet de la réunification et de la mondialisation?

\section{A. L'impact de la réunification : le nouvel attrait de l'ex-RDA}

Du temps de la division de l'Allemagne, le naturisme avait suivi des évolutions divergentes de part et d'autre de la frontière, la RFA reconstituant des structures naturistes comparables à celles des autres pays de l'Europe de l'ouest. En RDA, le nudisme s'était maintenu uniquement sous la forme d'une pratique sauvage en bord de lac ou de mer, sans cadre associatif, et avec une connotation de contestation politique évidente (film de G. Thiel, 1999). Dans les premières années du régime, de multiples incidents avaient opposé les nudistes aux forces de l'ordre, ces dernières étant fréquemment prises à partie par la foule des baigneurs à l'occasion de contraventions ou de tentatives de conduites au poste de police. Devant l'impossibilité d'endiguer le nudisme, les autorités avaient abandonné la répression en 1956 et finalement laissé les communes permettre la pratique en certains secteurs. Mais arguant du nombre insuffisant de plages officielles, les nudistes n'hésitaient pas à s'installer au-delà des panneaux de signalisation des secteurs autorisés. Ce nudisme proliférant et incontrôlé de la RDA, qui était un symbole de liberté individuelle en résistance au régime communiste, différait du naturisme institutionnalisé et plus clairement délimité de l'ouest.

Avec la chute du mur de Berlin, deux « mondes » naturistes sont entrés en collision. Si la disparition de la " frontière » a suscité un mélange de méfiance et d'enthousiasme de part et d'autre (Armbruster et Meinhof, 2003), il est indéniable qu'un effet de curiosité a attiré de nombreux touristes de l'ouest de l'Allemagne vers la côte de l'ex-RDA, qui leur était interdite pendant la période communiste. De fait, le tourisme estival s'y est fortement développé, et l'on a assisté depuis 1990 à un doublement de la capacité d'accueil du Land de Mecklembourg-Poméranie occidentale (Mangin, 2003). Dans ce même Land, les infrastructures hôtelières de bord de mer ont été largement réhabilitées, et trois campings naturistes reconnus par la F.K.K. se sont créés, tous dans la région intérieure des lacs du Mecklembourg: le camping de l'Useriner See, installé dans une forêt de pins en lisière du Parc National de Müritz, disposant d'une plage en bord de lac, et homologué 5 étoiles, est emblématique de ce tourisme en espace naturel alliant naturisme et confort. Sur le littoral, l'afflux de touristes originaires de l'ex-Rfa a eu des conséquences en chaîne sur le nudisme local. Dans un premier temps, un certain nombre d'entre eux ont été choqués par l'extension de la pratique nudiste hors des secteurs autorisés. Cela a conduit les autorités à augmenter les secteurs officiels pour essayer de mieux répartir les publics : ainsi sur l'île de Usedom, la plus grande plage 
nudiste officielle de l'île qui avait été ouverte en 1956 sur une longueur de 1,5 km s'est vue agrandie de $600 \mathrm{~m}$ en 2000, ce qui la rend aussi étendue que la plage "textile" voisine (Le Prioux, 2005). Malgré les efforts d'encadrement des autorités, le nudisme sauvage reste encore plus pratiqué dans les nouveaux Länder que dans ceux de l'ouest.

\section{B. L'inégale diffusion du modèle allemand à l'étranger}

A petite échelle, le nudo-naturisme allemand relève d'un modèle germanique caractéristique de l'Europe du nord protestante, associant un nudisme répandu dans les espaces publics et un nombre de centres de vacances naturistes somme toute modeste eu égard au public potentiel (Descamps, 1987). Ce dernier trait s'explique aussi par l'habitude de plusieurs dizaines de millions d'Allemands de passer leurs vacances d'été hors de leurs frontières, principalement dans des pays européens situés plus au sud. L'Allemagne constitue ainsi sur le continent européen le premier pôle de diffusion de touristes internationaux, avec une balance touristique très déficitaire: les entrées ne représentent que $35 \%$ des sorties de devises (Boyer, 2000). Ce tourisme allemand à l'étranger a d'autant plus servi de vecteur à la diffusion de la pratique nudiste qu'il est principalement balnéaire. Selon Mangin (2003), en Europe, les Allemands représentent environ $40 \%$ des clients étrangers des "destinations ensoleillées». La recherche de littoraux ensoleillés par les Allemands en vacances est ainsi un stéréotype sur lequel les romans de Michel Houellebecq jettent un regard ironique1. Les Allemands eux-mêmes n'ont-ils pas surnommé les Baléares le 17e Land, et sa capitale Palmenhausen ? Dans les centres naturistes français, si les Néerlandais préfèrent les petits centres des zones rurales de l'intérieur, les Allemands privilégient les gros centres littoraux de l'Aquitaine ou du Languedoc (Espaces, 1999) où ils représentent les principaux clients étrangers, et donc une cible de choix pour les responsables de centres ou de programmes immobiliers naturistes (photo 3). Toutefois, les directeurs de centres naturistes français notent un fléchissement de la fréquentation allemande, en partie liée à la montée de destinations naturistes plus récentes, principalement la Croatie (depuis la fin de la guerre de l'ex-Yougoslavie) et l'Espagne, offrant un hébergement plus récent et moins cher. 
Photo 3 : Panneau de promotion immobilière en allemand pour un nouveau lotissement naturiste à Port-Leucate (Aude). Juin 2006.

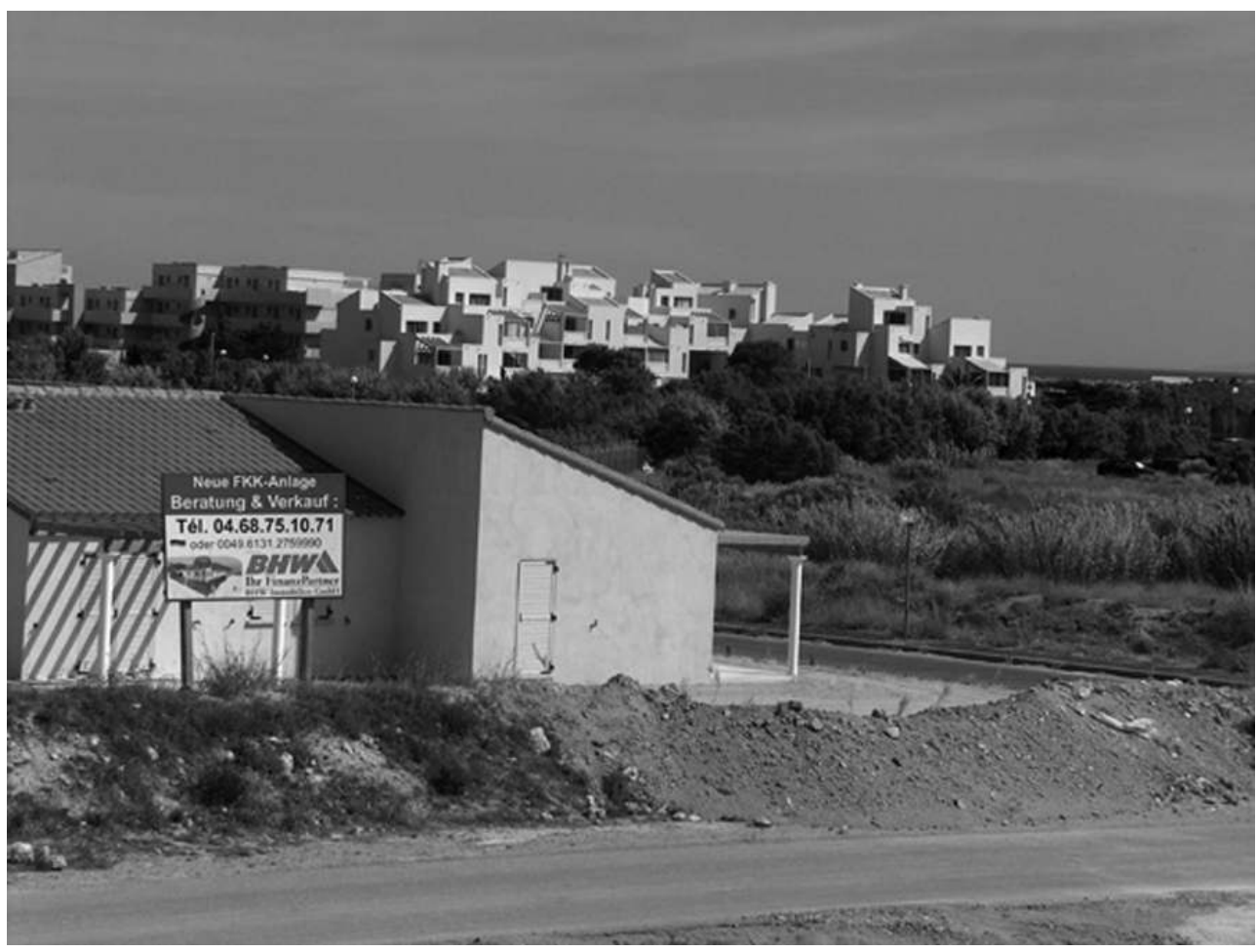

C'est aussi et plus encore par le nudisme de plage que les Allemands ont diffusé la pratique du nu auprès d'autres publics européens non naturistes, spécialement sur les rives nord de la Méditerranée (Jaurand, 2006). S. Villaret (2005) évoque les origines de la création du camping naturiste d'Agde par les frères Oltra sur une partie de leurs vignes en 1956 : l'idée leur était venue après avoir vu les soldats allemands se baigner nus sur les plages pendant l'Occupation, et tout désireux de revenir réitérer l'expérience une fois la guerre terminée. On sait que le camping Oltra a servi de point d'appui au lancement du futur quartier naturiste du Cap d'Agde. C'est ainsi qu'une conduite habituelle pour les Allemands a été transformée en affaire commerciale par des Français, illustration saisissante de la différence des naturismes des deux pays. L'Espagne, première destination de vacances des Allemands à l'étranger, montre un cas encore plus spectaculaire d'imitation d'une pratique nudiste importée. Le nouveau code pénal espagnol, datant de 1995, a aboli l'article 431 qui datait de la période franquiste et qui interdisait "toute manifestation de nudité » (ce qui incluait le maillot de bain 2 pièces pour les femmes et le slip pour les hommes, selon une définition de la nudité également partagée par des légistes musulmans...). Pour ne pas contrarier les habitudes des touristes allemands, aucune disposition restrictive n'a remplacé l'article 431, ce qui signifie en théorie que la nudité n'est pas hors-la-loi. Dans la pratique, le nudisme s'est encore davantage développé sur les plages espagnoles depuis une décennie: il est extrêmement fréquent sur toutes les plages isolées, surtout sur la côte méditerranéenne et aux îles Canaries, et de nombreux cas de plages nudistes urbaines existent, comme nous l'avons constaté à Barcelone ou Sitges. Le brassage des publics étranger et national sur ces plages témoigne de l'assimilation de la pratique nudiste par l'Espagne de la movida. La Turquie constitue une autre destination balnéaire prisée des Allemands : certains d'entre eux, par petits groupes, pratiquent le nudisme sur des portions de plages éloignées, voire sur des plages plus célèbres et fréquentées, mais à 
leur extrémité et à l'abri de parasols (cas observé à Ölüdeniz). Et alors que le guide de la FNI ne mentionne aucune rubrique sur la Turquie, la consultation d'un site internet allemand nous a informé de l'existence d'un petit centre naturiste en bord de mer, dans la région d'Antalya (site internet Nacktbaden). Ce cas unique en pays musulman témoignerait de l'officialisation d'une pratique touristique qui relève ici de la figure de l'enclave étrangère.

\section{Des évolutions convergentes en Allemagne et en France?}

Les pays germaniques du nord de l'Europe sont ainsi des foyers anciens de naturisme, et aussi des pôles de diffusion de la pratique à l'occasion des migrations vacancières vers les pays touristiques du sud de l'Europe. Par rapport à cette projection nord-sud, la France occupe une position unique, étant à la fois un autre berceau du mouvement naturiste au XXe siècle et le premier pays du monde pour les structures d'accueil naturistes. Cependant, plusieurs dynamiques communes affectent le naturisme des deux côtés du Rhin.

31 Dans les deux pays, le naturisme officiel connaît une désaffection du mouvement associatif et un certain vieillissement des militants. La montée de l'individualisme et des préoccupations commerciales aux dépens de l'idéal naturiste marquent le mouvement officiel, plus encore en France qu'en Allemagne. Des centres naturistes, spécialement les plus importants, font évoluer leur politique commerciale dans le sens d'une atténuation des contraintes et d'un élargissement de la clientèle: la licence fédérale n'est plus obligatoire pour y accéder, des publics non familiaux sont de plus en plus acceptés et la nudité devient le plus souvent facultative (clothe optionnal) sauf aux abords de la piscine.

Dans l'espace public, l'évolution de la place du nu semble plutôt en stagnation voire en recul sous l'effet de plusieurs processus. De nombreux signes d'une telle tendance sont repérables dans les espaces verts des villes d'Allemagne comme sur les plages françaises. Ainsi à Munich en 2002, Thomas Koster, le directeur du jardin anglais, constatait à l'échelle de plusieurs années une forte chute du nombre de nudistes : par une journée chaude d'été, il évaluait leur nombre à 1000 environ contre 14000 par le passé (Kirschbaum E., 2002). Il explique ce phénomène par le retour d'un certain conservatisme et la nouvelle culture dominante des jeunes, très portée sur la mise en scène du corps par le vêtement, le logo ou le piercing, et étrangère au nu qui était symbole de rébellion dans les années 60-70 marquées par la révolution sexuelle et le mouvement hippie. Il ajoute l'importance du voyeurisme de la part d'étrangers, touristes ou immigrés, provenant de pays où ce type de nudité publique n'existe pas et s'installant tout habillés au milieu des nudistes. Ce second facteur, important pour la plupart des sites nudistes en espace public, décourage surtout les femmes. Il s'ensuit une masculinisation de la pratique nudiste aisée à constater dans les deux pays, avec une surreprésentation des gays qui tendent à déployer leurs propres codes de conduite, notamment par une sexualisation de l'espace public. Le caractère «non familial » de la pratique nudiste a d'ailleurs conduit plusieurs municipalités du littoral français à mettre fin aux arrêtés d'autorisation ou à la tolérance du nudisme sur plusieurs plages (Hyères, Zuydcotte, Wissant...) en faisant appel aux forces de l'ordre pour verbaliser voire arrêter les contrevenants. Les pressions foncières sur le littoral, plus fortes en 
France qu'en Allemagne, spécialement en Méditerranée, expliquent aussi la disparition d'autres plages naturistes officielles (Fos-sur-mer).

Au final, la moindre observance de la nudité dans les centres naturistes et l'invasion des plages nudistes par de nombreux «textiles » rompent la séparation étanche entre l'univers clos du centre et l'espace public. Ce faisant, le modèle allemand fondé sur une banalisation de la nudité au milieu des autres dans l'indifférence tend à la fois à se diffuser à l'étranger, avec des réactions négatives, et à marquer un certain recul numérique en Allemagne.

Pour conclure, il semble indéniable que par le brassage des touristes de toutes nationalités dans les pays d'Europe du sud et en France, et l'effet d'imitation de pratiques de nudité habituelles chez les Allemands, le naturisme et le nudisme d'origine germanique ont conquis des publics et investi des territoires à l'étranger. Le spectacle de la nudité banalisée sur de nombreuses plages de l'Espagne, de Croatie, d'Aquitaine ou du Languedoc, constitue un signe d'imitation (mais non de transfert tel quel) du modèle allemand au-delà des frontières du pays. L'exception française réside dans la combinaison d'un naturisme endogène et exogène, intégré au tourisme balnéaire de masse, et accompagnée de réalisations urbaines uniques au monde (Le Cap d'Agde, Port-Leucate). Mais au-delà de la relative banalisation du signifiant nu en des lieux publics déterminés, il convient de s'interroger sur le signifié culturel d'une telle manifestation. De ce point de vue, le modèle naturiste allemand nous semble conserver toute son originalité et sa profondeur, et sa diffusion s'est faite sur un mode édulcoré et tronqué (la pratique nudiste sans l'arrière-plan philosophique ou culturel). Comme dans toute l'Europe protestante du nord, le nu germanique est à la fois ancien, collectif, mixte, désexualisé et pratiqué en espace de nature le plus souvent possible en toutes saisons. Il se fonde sur l'idée d'une double harmonie qui fonde l'éthique naturiste : des êtres humains avec une nature incarnation du divin, et à ce titre objet d'un respect attentif, et des individus égaux entre eux en droit, en dignité sans préjugé d'ordre esthétique ou connotation sexuelle. Les motivations à la pratique du nu sont avant tout la santé, le sport et le rapprochement avec la nature (Schoelzke, 2006). En Allemagne, la nature est ainsi pratiquée et vécue plus intimement, fréquemment, jusqu'au cœur de villes dans lesquelles elle est intégrée : une même pratique nudiste est ainsi acceptée en bord de mer ou en jardin public, preuve qu'elle constitue plus une habitude culturelle indépendante de la saison et des lieux touristiques. En France comme dans d'autres pays latins, la nature "sauvage " est vécue sur le mode de la rupture saisonnière et spatiale: d'où le succès de centres naturistes de vacances qui offrent un dépaysement par rapport au mode de vie habituel, tandis que le nudisme de plage y revêt une dimension communautaire et sexuelle plus affirmée.

\section{BIBLIOGRAPHIE}

ARMBRUSTER H., MEINHOF U. H. (2003). - « Vécu et perception de la frontière entre la RDA et la RFA », Revue Géographique de l'Est, Tome XLIII, n² 4, p. 151-160. 
BARTHE F. (2001). - « Géographie du naturisme : à la recherche de l'éden », Géographie et Cultures, $\mathrm{n}^{\circ} 37$, p. 37-58.

BARTHE-DELOIZY F. (2003). - « Le naturisme : des cures atmosphériques au tourisme durable », Communications, Tome 74, p. 49-64.

BAUBEROT A. (2004). - Histoire du Naturisme : le mythe du retour à la nature, Rennes, Presses Universitaires de Rennes, $348 \mathrm{p}$.

BOULAND J.L. (1997). - Tout en nu de A à Z, Paris, Encre, 249 p.

BOYER J.C. (1996). - « La frontière entre protestantisme et catholicisme en Europe », Annales de Géographie, Tome 105, n 587, p. 119-140.

BOYER J.C. (2000). - Géographie humaine de l'Allemagne, Paris, Colin, 2e ed., 206 p.

ClaVAl P. (1995). - La géographie culturelle, Paris, Nathan, 384 p.

CLUET M. (1999). - La « libre culture » : le mouvement nudiste en Allemagne depuis ses origines au seuil du XXe siècle jusqu'à l'arrivée de Hitler au pouvoir (1905-1933), Université de Paris IV, Thèse de doctorat d'Etat inédite, 3 tomes, $1145 \mathrm{p}$.

DESCAMPS M.A. (1987). - Vivre nu : psychosociologie du naturisme, Paris, Trismégiste, 230 p.

Espaces (1999). - Dossier « naturisme », $\mathrm{n}^{\circ} 162, \mathrm{p} .13-33$.

Fédération Naturiste Internationale (2003). - Guide naturiste mondial, Anvers, Warlich, 634 p.

JAURAND E., LUZE H. de (2004). - « Ces plages où les genres s'affichent ? Les territoires du nu sur la Côte d'Azur ». In : Bard C. - dir. - Le genre des territoires : masculin, féminin, neutre, Angers, Presses de l'Université d'Angers, p. 227-240.

JAURAND E. (2006). - « Le tourisme naturiste en Méditerranée : entre interface et choc des civilisations? ", Bulletin de l'Association des Géographes Français, Tome 83, n 3, p. 331-340. KIRSCHBAUM E. (2002). - Uhr Nackte kehren Englischem Garten in München den Rücken, http://perso.orange.fr/plagenat/resultat/etranger/rem_allemagne.htm, 12 septembre 2006 KÖNIG O. (1990). - Nacktheit : Soziale Normierung und Moral, Opladen, Westdeutscher Verlag, 400 p. LE PRIOUX C. (2005). - Avec ou sans maillot sur les plages allemandes, http://www.vivrenu.com/article.php?sid=560, 23 mai 2006

LOZATO-GIOTART J.P. (1993). - Géographie du tourisme, Paris, Masson, 4e ed., 309 p.

MANGIN C. (2003). - L'Allemagne, Paris, Belin, 191 p.

PITTE J.-R. (1999). — « La vision catholique de la nature et de l'environnement et ses conséquences sur l'aménagement de l'espace ». In : Pitte J.R. et Sanguin A.L. - dir. -, Géographie et liberté, Mélanges en hommage à Paul Claval, Paris, L'Harmattan, p. 659-669.

ROBIC M.-C. - dir. (1992). - Du milieu à l'environnement. Pratiques et représentations du rapport homme/nature depuis la Renaissance, Paris, Economica, 343 p.

Ross C. (2005). - Naked Germany : health, race and the nation, Oxford/New York, Berg, 239 p. ROYER L.C. (1929). - Au pays des hommes nus, Paris, Editions de Paris, 216 p.

schoelzKe V.M. (2006). - « Nudité "naturelle" et perceptions "culturelles” », La Vie au Soleil, n¹08, pp. 53-54. 
TOEPFER K. (1997). - Empire of Ecstasy : nudity and movement in German body culture (1910-1935),

Berkeley/Los Angeles, University of California Press, 422 p.

VIARD J. (1990). - Le Tiers-Espace : essai sur la nature, Paris, Méridiens Klincksiek, 152 p.

VILLARET S. (2005). - Histoire du naturisme en France depuis le siècle des Lumières, Paris, Vuibert, $378 \mathrm{p}$.

WEDEMEYER M. (2001). - « “Unter allen Umständen bade man ohne Kleider...” : Zur Geschichte des Nacktbadens auf Sylt ». In : Jessel H., Des Grosse Sylt Buch, Hamburg, Ellert und Richter, p. 182-185.

\section{Sites internet consultés}

Fédération allemande de naturisme, http://www.dfk.org, 4 avril 2006

Fédération française de naturisme, http://www.ffn-naturisme. com, 23 mai 2006

Fédération internationale de naturisme, http://www.inffni.org, 12 septembre 2006

Fédération néerlandaise de naturisme, http://www.nfn.nl, 23 mai 2006

Fédération québecquoise, http://www.fqn.qc.ca, 4 avril 2006.

Nacktbaden, http://www.nacktbaden.de, 12 septembre 2006.

\section{Filmographie}

THIEL G. (1999). - Nu et libre. Histoire du naturisme. Documentaire, régulièrement diffusé sur Arte (notamment les 7/11/2001 et 11/5/2005), 55 minutes.

SALIS R. (1998). - Vivre nu. A la recherche du paradis perdu. Documentaire, Eden films, $1 \mathrm{~h} 44$, disponible en DVD.

\section{RÉSUMÉS}

Le naturisme constitue une pratique de nature collective dans un état de nudité associée au respect des autres et de l'environnement. Il est anciennement développé en Allemagne et dans les pays protestants d'Europe du nord où la nature est mythifiée. Le nudisme y est banalisé jusque dans l'espace public (plages et espaces verts urbains). En Allemagne, les centres naturistes sont répartis de façon assez homogène, sauf à l'Est, pour des loisirs nus toute l'année, alors qu'en France ils se concentrent dans les régions touristiques ensoleillées. Les dynamiques spatiales récentes du naturisme en Europe montrent un relatif effacement des spécificités nationales par diffusion du modèle allemand.

Naturism is a form of collective practice of nature in a state of nudity associated to respect for the environment and other people. It developed early in Germany and in Protestant countries of northern Europe where nature has a mythic value. Nudism has become common even in public spaces such as beaches or urban parks. In Germany, naturist centres exist in all the country, though there are fewer in the East, so nude leisure may be enjoyed throughout the year. In France, they are concentrated in touristic, sunny regions. However, recent trends of naturism show a spread of the German model in Europe.

Die Freikörperkultur ist eine kollektive Freiluftaktivität, die Nacktheit mit dem Respekt vor Anderen und vor der Umwelt assoziiert. Sie entstand schon früh in Deutschland und den protestantischen Ländern Nordeuropas, wo die Natur mythologisiert ist. Dort ist Nudismus bis in 
öffentliche Räume (Strände und städtische Grünanlagen) hinein verbreitet. In Deutschland sind die Zentren der Freikörperkultur gleichmäßig verteilt, außer im Osten, so dass ihre Ausübung ganzjährig möglich ist. In Frankreich hingegen sind sie auf die sonnenreichen Tourismusregionen beschränkt. Die jüngere räumliche Dynamik von FKK in Europa zeigt eine tendenzielle Verwischung der nationalen Besonderheiten durch Ausbreitung des deutschen Modells.

\section{INDEX}

Keywords : naturism, nudism, tourism, nature, beach, public space, Germany, France Schlüsselwörter : Freikörperkultur, Nudismus, Fremdenverkehr, Natur, Strand, Öffentlicher Raum, Deutschland, Frankreich

Mots-clés : naturisme, nudisme, tourisme, nature, plage, espace public, Allemagne, France

\section{AUTEUR}

\section{EMMANUEL JAURAND}

Département de Géographie - U.F.R. de Lettres et Sciences Humaines - Université de Paris XII 61, avenue du Général de Gaulle - F-94010 Créteil Cedex 